\title{
DRAFT \\ Bubble properties of heterogeneous bubbly flows in a square bubble column
}

\author{
Wei Bai, Niels G. Deen and J.A.M. Kuipers \\ Fundamentals of Chemical Reaction Engineering, Faculty of Science and Engineering, \\ Institute of Mechanics, Processes and Control Twente (IMPACT), \\ University of Twente, 7500 AE Enschede, the Netherlands, N.G.Deen@utwente.nl
}

\begin{abstract}
The present work focuses on the measurements of bubble properties in heterogeneous bubbly flows in a square bubble column. A four-point optical fibre probe was used for this purpose. The accuracy and intrusive effect of the optical probe was investigated first. The results show that the optical probe underestimates bubble properties, such as, bubble velocity, local void fraction. The presence of the probe in the bubble column influences the local flow conditions. Particularly, when the probe is placed close to the liquid surface, this influence is more pronounced. Furthermore, two methods determining interfacial area were compared. The results from both methods agree quite well at low superficial gas velocity, whereas large discrepancies are found when superficial gas velocity increases. Finally, effect of column height on bubble properties was studied. No significant difference was found for the three investigated columns.
\end{abstract}

Keywords: Bubble column, four-point optical fibre probe, superficial gas velocity, bubble velocity, void fraction

\section{Introduction}

Bubble columns are intensively utilized as multiphase contactors and reactors in chemical, petrochemical, biochemical and metallurgical industrials. There are several advantages during operation and maintenance, such as high heat and mass transfer rates, compactness and low operating and maintenance costs.

For the operation of two-phase flows in bubble columns, insight in primary parameters such as bubble size, shape and velocity as well as void fraction is essential. For example, bubble characteristics play a key role in the performances of heat and mass transfer in bubble column reactors.

At high void fractions, the flow system becomes opaque, ruling out camera techniques. Optical fibre probes (Cartellier 1990, Mudde 2001) have the advantage of low cost, simplicity of setup and easy interpretation of the results. With an optical fibre probe, the measurement of local void fraction becomes possible. Furthermore, using a four-point optical probe, properties of bubbles can also be studied, such as bubble velocity and bubble size.
Although a lot of research has been carried out in two-phase flows in the past decades, effort to enhance the understanding of the hydrodynamical behaviour of bubbles is necessary. For this purpose, in this work we study bubbly flows inside a square column with superficial gas velocities ranging from 0.005 $\mathrm{m} / \mathrm{s}$ to $0.1 \mathrm{~m} / \mathrm{s}$. By using a four-point optical probe, some details about bubble properties, such as bubble velocity and local void fraction can be revealed.

\section{Experimental setup}

The experiments were carried out in a square column with a cross-sectional area $(W \times D)$ of $0.15 \times 0.15 \mathrm{~m}^{2}$. A perforated plate with 49 holes of $1 \mathrm{~mm}$ in diameter is used as a gas sparger at the bottom of the column. These holes are located evenly at a square pitch of $6.25 \mathrm{~mm}$ in the central region of the perforated plate. Demineralized water is used as liquid phase and the gas phase is air.

A sketch of the experimental setup is shown in Fig. 1. A four-point optical probe was inserted through one of the side walls, which is made of steel. 
A differential pressure transducer $D P$ was used to measure the differential pressure in the column.

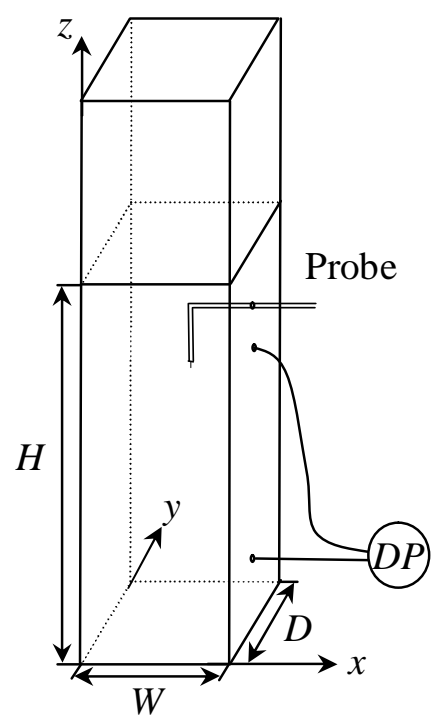

Fig. 1. Sketch of experimental setup.

During the measurements, three different column aspect ratios $(H / W=3, H / W=6, H / W=9)$ were investigated.

Nearly all the measurements were taken at a height of $339 \mathrm{~mm}$ which is at $z / H=0.75,0.38$ and 0.25 for the respective column heights. The probe was moved in the middle plane horizontally.

\section{Data processing}

\section{Interpretation of probe data}

Optical probes have been used to study bubble properties in two-phase flows for decades. The principle of optical probe is based on different refractive indices of light in the two phases. With sufficient signal to noise ratio, the two phases can be distinguished. Consequently, by using a separation method, the gas phase can be easily retrieved from the output signal. This makes it possible to deduce bubble properties, i.e. local void fraction. Furthermore, it is also possible to obtain the bubble velocity when multiple probe tips are used.

As an intrusive instrument, however, the presence of probe influences the local flow conditions and thus, the measurements of the optical probe. This intrusive effect will be discussed in the paper.

The four-point optical probe has three tips of the same length that form an equivalent angle. The fourth tip is positioned in the center between the other three tips. The radial distance $d_{p}=0.5 \mathrm{~mm}$ and the vertical distance is $\Delta s=1.4 \mathrm{~mm}$. The configuration of the probe is shown in Fig. 2.
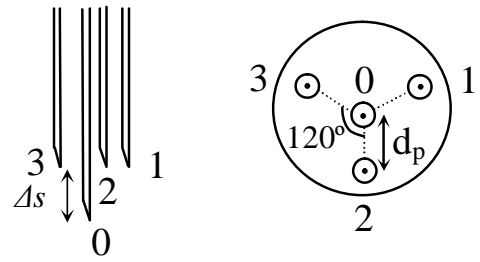

Fig. 2. Configuration of four-point optical probe.

The interpretation of the probe signal is based on the method used by Julia et al. (2005) and Harteveld (2005). That is, the gas phase is determined with the following threshold: $V_{T}=V_{L}+0.1\left(V_{G}-V_{L}\right)$. Where $V_{T}$ is the threshold, $V_{L}$ is signal level of liquid phase and $V_{G}$ is signal level of gas phase.

The local void fraction can be expressed as the ratio between the accumulated bubble sampling time and the total sampling time (Cartellier, 1990).

$$
\alpha=\frac{\Sigma t}{T}
$$

In this work, the local void fraction is determined by the central tip (tip 0 ).

The bubble velocity can be determined from the distance between the central tip and the other three tips and the average time difference that the bubble hits the central tip and the other three tips:

$$
u=\frac{\Delta s}{\Delta t}=\frac{\Delta s}{\frac{1}{3} \Sigma \Delta t_{i}}
$$

where $\Delta t_{i}=t_{i}-t_{0} \quad(i=1,2,3) \quad$ is the time difference of bubble between the central tip (tip $0)$ and the three other tips.

During the calculation of the bubble velocity, 
only those bubbles that satisfy the following criterion of bubble selection are selected.

$$
\left(\frac{\Delta t_{i}-\Delta t}{\Delta t}\right)<\beta
$$

This criterion has a strong effect on the fraction of valid bubbles and on the accuracy of the results. Mudde and Saito (2001) found the best agreement between velocities measured with a probe and measured with a camera for $\beta=0.08$. With such choice, however, the fraction of valid bubbles would be rather low. Therefore, $\beta=0.20$ was used in their experiments and the results were acceptable. Fortunati et al. (2002) studied the sensitivity of the bubble selection criterion by analysing the probability density function of bubble velocity and found that the probability density function of bubble velocity does not change considerably by increasing $\beta$ up to 0.25 . In the present paper, in order to increase the valid bubble fraction, $\beta=0.40$ was used. Consequently the valid bubble fraction increases from about $20 \%$ to $37 \%$ when $\beta$ is increased from 0.25 to 0.40 . The resulting deviation of bubble velocity and mean bubble chord length is about respectively $2 \%$ and $1 \%$.

During measurements, at least 1500 bubbles selected from the criterion mentioned above are used to calculate the average bubble velocity at each location. Particularly, in cases of high superficial gas velocity, more than 3000 bubbles are selected, i.e. above 10,000 bubbles are used in case of superficial gas velocity $u_{g}=$ $0.6 \mathrm{~m} / \mathrm{s}$.

\section{Differential pressure transducer}

By measuring the differential pressure $\Delta P$ over a certain height $\Delta z$ before and after injecting gas into column, one can calculate gas holdup $\bar{\alpha}$ with the following equation.

$$
\bar{\alpha}=\frac{\Delta P}{\rho g \Delta z}
$$

It is assumed that the mass of the gas phase is negligible compared to that of the liquid phase.

\section{Interfacial area calculation}

In two-phase flow systems, the interfacial area concentration is of importance. Assuming spherical bubbles, the interfacial area concentration is related to the gas holdup $\bar{\alpha}$ and the sauter mean bubble diameter $d_{s}$.

$$
a=\frac{6 \bar{\alpha}}{d_{s}}
$$

By using a four-point optical probe, however, one can only obtain chord lengths of bubbles instead of diameters of bubbles. Some researchers tried to find a relationship between the chord length distribution and the bubble size distribution in a statistical way (Lim 1990, Liu 1995, 1998, etc). Determination of the bubble size distribution from the chord length distribution is a typical inversion problem. By using some assumptions, such as bubble shape and angle of attack, use of backward transformation to determine the bubble size distribution is possible. In the present study, the bubble size distribution is not considered. Only the mean values of bubbles are investigated. Therefore, according to Liu (1995), the equivalent bubble diameter $d_{e}$ can be estimated with the following formula by assuming bubbles approaching the probe with a zero angle of attack and ellipsoidal bubbles.

$$
d_{e}=\frac{3}{2} R_{z} E^{-2 / 3}
$$

where $E$ is aspect ratio of bubble and $R_{z}$ is the mean chord length.

When the bubble aspect ratio of bubble is known, one is able to determine the equivalent bubble diameter and thus, the interfacial area.

The aspect ratio of the bubble, however, is difficult to be determined because of the flow complexity. Dijkhuizen (2008) investigated aspect ratio of single bubble in stagnant liquids numerically and experimentally. He found a bubble aspect ratio of around 0.7 when $d_{e}=0.004 \mathrm{~m}$ in his experiment. In the present work, by considering influence of the complex flow on bubble shape, an estimation 
of $E=0.7$ is adopted to calculate bubble size.

Kataoka (1986) derived the following formula for local time-averaged interfacial area.

$$
\bar{a}(x, y, z)=\frac{1}{T} \sum \frac{1}{|v| \cos \varphi}
$$

where $T$ is total measurement time and $\varphi$ is the angle between the interface velocity vector $\dot{v}$ and the direction of the surface normal vector at $(x, y, z)$.

By assuming spherical bubbles and the probe passes every part of the bubble with an equal probability, the formula can be simplified as follows.

$$
\bar{a}(x, y, z)=4 N \frac{\overline{1}}{|\vec{v}|}
$$

where $N$ is the bubble frequency, i.e. the number of bubbles hitting the probe per unit time.

In the present work, both methods for determining the interfacial area are adopted.

\section{Results and discussions}

\section{Intrusive effect of probe}

There is no doubt that the optical probe, as an intrusive instrument, locally disturbs the flow field and thus, the bubble properties obtained from the probe. To investigate this intrusive effect and verify the feasibility of probe, an extra measurement was performed. The bubble properties at three different depths $(y / D=0.5, \quad y / D=0.75, \quad y / D=0.875) \quad$ were measured with the four-point optical probe. The small column $(H / W=3)$ was used. The optical probe was inserted through the side wall at nearly half height $(z / H=0.55)$ of the column. Two cases with different superficial gas velocities $\left(u_{g}=0.02 \mathrm{~m} / \mathrm{s}, \quad u_{g}=0.1 \mathrm{~m} / \mathrm{s}\right)$ were considered.

When the optical probe is used to obtain values for local void fraction $\alpha$ and bubble velocity $u$ at different positions in one horizontal plane, the overall gas flow rate $Q$ can be computed with the following formula and compared with that from the flow meter to verify the accuracy of the optical probe.

$$
Q=\int_{A} \alpha(x, y) u(x, y) d A
$$

where $A$ is cross-sectional area of the column. By assuming symmetry of the flow, the total gas flow rate is obtained from calculating gas flow rate over the half area of cross section and/or a quarter of the cross sectional area.

Furthermore, surface-averaged void fraction can be calculated from the following formula.

$$
\bar{\alpha}=\frac{1}{A} \int_{A} \alpha(x, y) d A
$$

The surface-averaged void fraction can also be compared with the gas holdup obtained from the differential pressure transducer.

The comparisons of both gas flow rate and gas holdup are listed in table 1 .

Table 1

Comparisons of both gas flow rate and gas holdup

\begin{tabular}{|c|c|c|c|c|}
\hline \multirow{2}{*}{ Method } & \multicolumn{2}{|c|}{$u_{g}=0.02 \mathrm{~m} / \mathrm{s}$} & \multicolumn{2}{c|}{$u_{g}=0.1 \mathrm{~m} / \mathrm{s}$} \\
\cline { 2 - 5 } & $Q\left[\mathrm{~m}^{3} / \mathrm{s}\right]$ & $\bar{\alpha}[-]$ & $Q\left[\mathrm{~m}^{3} / \mathrm{s}\right]$ & $\bar{\alpha}[-]$ \\
\hline Measured & $4.49 \cdot 10^{-4}$ & 0.026 & $2.25 \cdot 10^{-3}$ & 0.11 \\
\hline Probe, $A / 2$ & $2.98 \cdot 10^{-4}$ & 0.023 & $1.53 \cdot 10^{-3}$ & 0.08 \\
\hline Probe, $A / 4$ & $3.13 \cdot 10^{-4}$ & 0.024 & $1.58 \cdot 10^{-3}$ & 0.09 \\
\hline
\end{tabular}

It can be seen that the gas flow rates obtained from the optical probe are less than those from the flow meter. The gas flow rate from the optical probe differs about $30 \%$ from that obtained with the flow meter. The gas holdup from the optical probe has a much smaller deviation compared with results from the differential pressure transducer.

The reasons for the observed discrepancies may be various. Besides the assumption of symmetry of flows inside column, the 
processing method of optical probe rules out many bubbles that are rising inside the column at a large angle of attack and that do not hit the four tips of the probe. Furthermore, the intrusive effect of probe is also one of the reasons. From Fig. 3, it can be found that in the locations $(x / W>0.5)$ some of the local void fractions are slightly smaller than those in corresponding locations $(x / W<0.5)$. Since the probe is inserted through the side wall at $x / W$ $=1$, the support of probe influences the flow there.

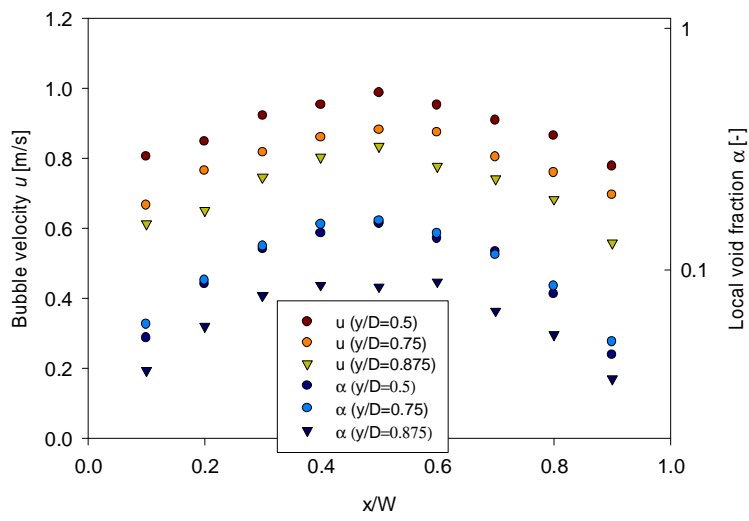

Fig. 3. Distributions of bubble velocity and local void fraction $\left(u_{g}=0.1 \mathrm{~m} / \mathrm{s}, z / H=0.38, H / W=6\right)$.

\section{Comparisons of interfacial area}

The two methods to calculate the interfacial area from the optical probe mentioned above are compared. The comparisons are taken in a column $(H / W=6)$ with three different superficial gas velocities $\left(u_{g}=0.005 \mathrm{~m} / \mathrm{s}\right.$, $u_{g}=0.04 \mathrm{~m} / \mathrm{s}, u_{g}=0.1 \mathrm{~m} / \mathrm{s}$ ).

The results are shown in Fig. 4. It can be seen that there is no significant difference between the two methods of determining the interfacial area from the optical probe when the superficial gas velocity is low $\left(u_{g}=0.005 \mathrm{~m} / \mathrm{s}\right)$. However, one can find some large discrepancies when superficial gas velocity increases. Due to intensive interactions between bubble and bubble, coalescences of bubbles become frequent at high superficial gas velocity. Big bubbles are no longer in ellipsoid shape. Therefore, the assumption of ellipsoid shape of bubble would not be feasible any more. And the estimation of $E=0.7$ would not be correct.

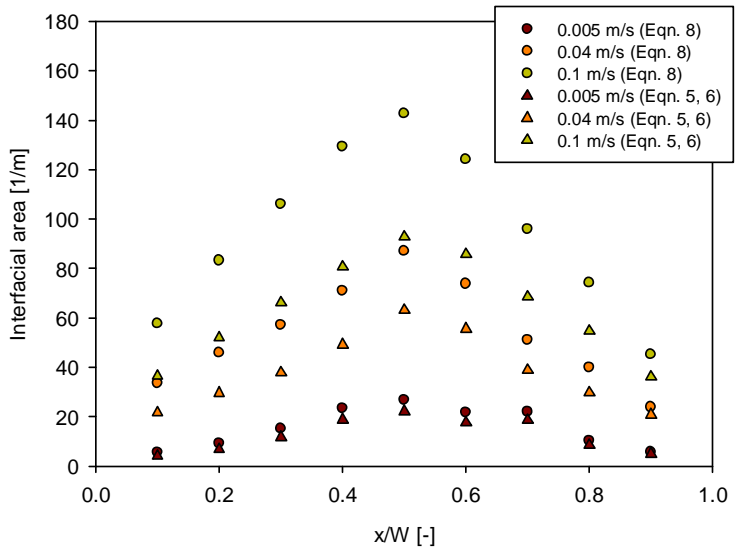

Fig. 4. Distributions of interfacial area $(z / H=0.38$, $H / W=6)$.

\section{Effect of column height}

The effect of column height on the bubble properties, such as bubble velocity, local void fraction, interfacial area and equivalent diameter, will now be discussed. Results measured at a height of $339 \mathrm{~mm}$ in the three columns are shown in Fig. 5. In this figure, the interfacial area is calculated from Eq. 8 and the equivalent bubble diameters are obtained from Eq. 5.

From the plots, it can be seen that the column height does not have a significant effect on the bubble properties inside the column. For example, at a superficial gas velocity $u_{g}=0.005$ $\mathrm{m} / \mathrm{s}$, the bubble velocity and the local void fraction in the three columns have similar profiles. And the profiles are more or less flat. This is caused by the meandering motion of a bubble plume inside column.

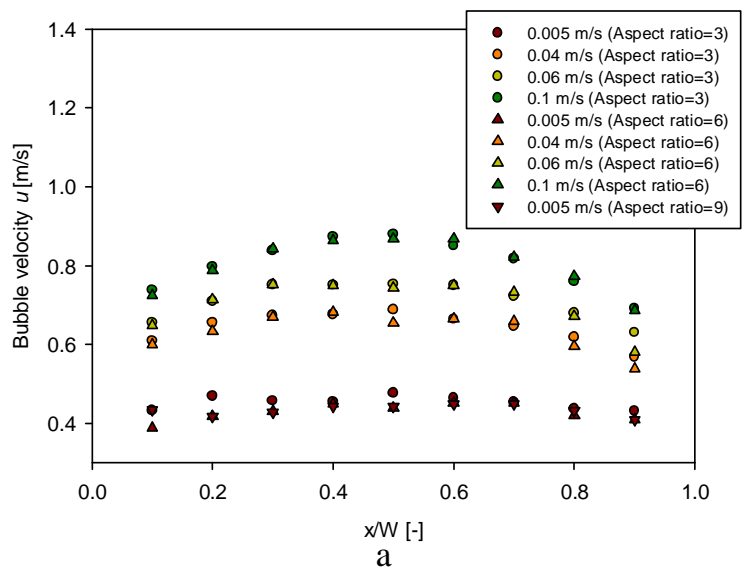



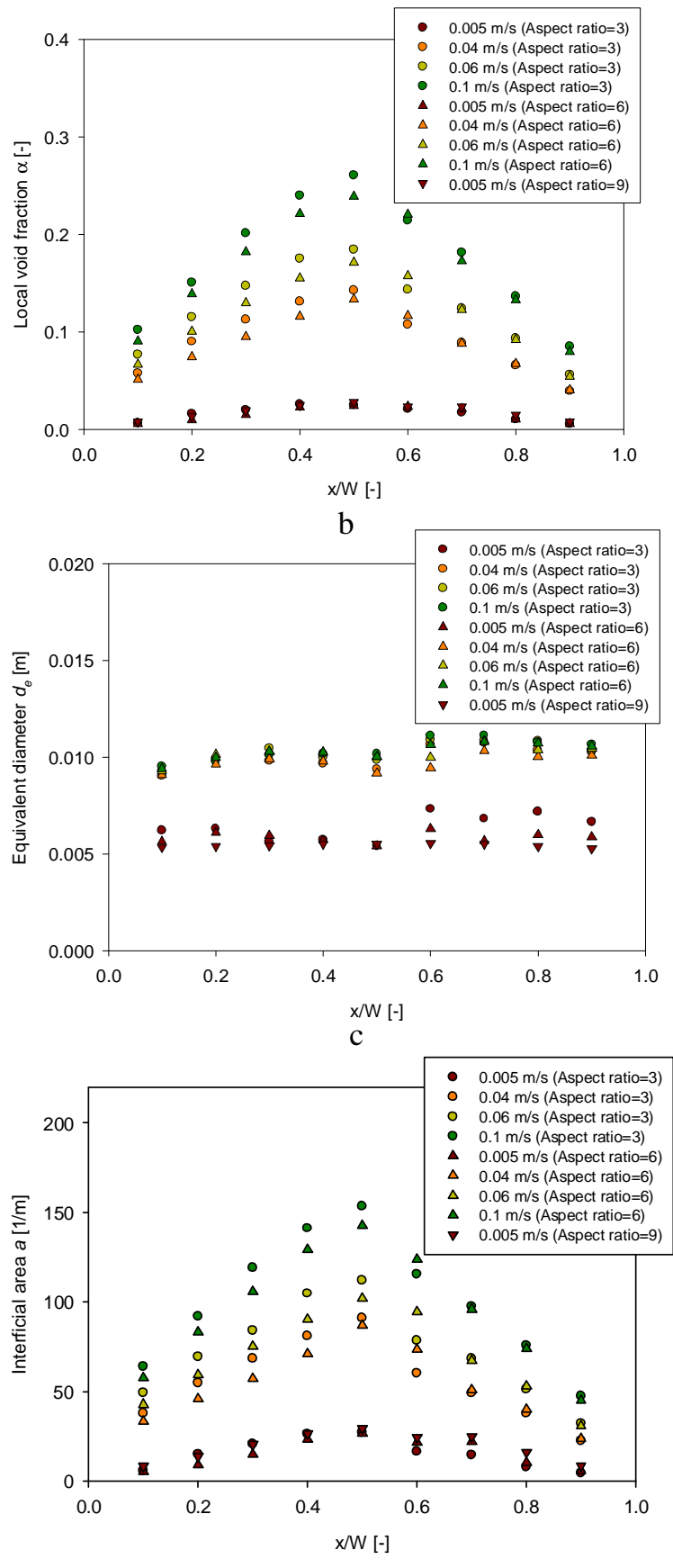

d

Fig. 5. Distributions of bubble properties with different superfacial gas velocities and column heights $(z=339 \mathrm{~mm})$. (a) Bubble velocity. (b) Local void fraction. (c) Equivalent diameter.

(d) Interfacial area

When increasing the superficial gas velocity, the distributions of bubble velocity in two columns $(H / W=3$ and 6$)$ also have similar patterns. However, the local void fraction and interfacial area in the small column are slightly higher than those in the large column. In the small column, the position of the probe is quite close to the surface of liquid $(z / H=0.75)$, whereas, in the large column, the probe is far away from the liquid surface $(z / H=0.38)$. Liquid circulation near the free surface may be stronger in the small column than in the large column. Thus, bubbles may touch the probe more frequently.

Furthermore, it is found that the equivalent bubble diameters are more or less the same in the three columns. When the superficial gas velocity increases, the equivalent bubble diameter also increases. When the superficial gas velocity is larger than $0.04 \mathrm{~m} / \mathrm{s}$, however, the equivalent bubble diameter only changes a little.

In the plots, it can be found that bubble properties in the region where $x / W>0.5$ are smaller than those in the region where $x / W<0.5$, which is probably due to the probe disturbance.

\section{Conclusions}

In the present work, we studied bubble properties in bubble column with different superficial gas velocities. A four-point optical fibre probe was used to measure the relevant quantities, such as bubble velocity and local void fraction. The accuracy of the optical probe was studied. The results deduced from the optical probe, such as gas flow rate and areaaveraged void fraction are compared with those obtained from a flow meter and a differential pressure transducer. Discrepancy between results from the optical probe and the flow meter and the differential pressure transducer was found, which is probably due to the intrusive effect of the probe.

Two methods for determining interfacial area were compared. They agree with each other quite well at low superficial gas velocity. However, discrepancies are found when superficial gas velocity increases.

Furthermore, the effect of column height on the bubble properties was investigated. It was found that the flow hardly depends on the column aspect ratio except for the local void 
fraction, which is slightly higher for the low aspect ratio.

\section{References}

Cartellier, A., 1990, Optical probes for local void fraction measurements: Characterization of performance, Rev. Sci. Instrum, 62(2), 874-886.

Dijkhuizen, W., 2008, Derivation of closures for bubbly flows using direct numerical simulations, $\mathrm{PhD}$ thesis, University of Twente.

Julia, J.E. et al., 2005, On the accuracy of the void fraction measurements using optical probes in bubbly flows, Rev. Sci. Instrum, 76, 035103-1-13.

Fortunati, R. et al., 2002, Accuracy and feasibility of bubble dynamic measurements with four point optical fiber probes, Proc. $11^{\text {th }}$ Symposium on Application of Laser Technique to Fluid Mechanics, Lisbon.

Harteveld, W., 2005, Bubble columns: structures or stability? $\mathrm{PhD}$ thesis, Technology University of Delft.

Kataota, I., Ishii, M. and Serizawa, A., 1986, Local formulation and measurements of interfacial area concentration in two-phase flow, Int. J. Multiphase Flow, 12(4), 505-529.

Lim, K. S. and Agarwal, Pradeep K., 1990, Conversion of pierced lengths measured at a probe to bubble size measures: an assessment of geometrical probability approach and bubble shape models, Powder Technology, 63, 205-219.

Liu, W. and Clark, N. N., 1995, Relationships between distributions of chord lengths and distributions of bubble sizes including their statistical parameters, Int. J. Multiphase Flow, 21(6), 1073-1089.

Liu, W. and Clark. Nigel N. and Karamavruc, Ali Ihsan, 1998, Relationship between bubble size distributions and chord-length distribution in heterogeneously bubbling systems, Chemical Engineering Science, 53(6), 1267-1276.

Mudde, Robert F. and Saito Takayuki, 2001, Hydrodynamical similarities between bubble column and bubbly pipe flow, J. Fluid Mech, 437, 203-228. 